\title{
THERMAL STIMULUS METHOD APPLIED TO INSULATING LIQUIDS: NUMERICAL ANALYSIS AND DISCUSSION OF THE THERMAL CONVECTION EFFECTS
}

\author{
Xavier SIDAMBAROMPOULÉ, Petru NOTINGHER, Jean-Charles LAURENTIE, \\ Thierry PAILLAT*, Paul LEBLANC* \\ $\underline{\text { Xavier.Sidambarompooule@ies.univ-montp2.fr, Petru.Notingher@umontpellier.fr }}$ \\ Université de Montpellier, CNRS, Montpellier, France \\ *Université de Poitiers, CNRS, Poitiers, France
}

\begin{abstract}
$\underline{\text { Abstract }}$
Non-destructive thermal techniques have been developed and extensively used during the last decades for space charge measurements in solid dielectrics. These methods are based on measuring and analyzing a transient capacitive current or voltage due to the redistribution of influence charges when a heat flow induced by an external thermal stimulus diffuses through the sample. If this principle is à priori usable on liquids, its application to such materials and appropriate interpretation of the measured signals require assessing and identifying the effects of thermal convection, which is likely to appear when heat diffuses in a liquid medium. This issue is addressed in the present work. Transient current responses of dielectric liquids with two different viscosities containing interface charges are computed numerically in the absence and in the presence of thermal convection when thermal stimuli of amplitudes up to $20 \mathrm{~K}$ are applied. The effects of the thermal convection are analyzed and guidelines for reducing them are proposed.
\end{abstract}

Keywords: space charge, thermal step method, dielectric, liquid, electric field, electrical double layer 


\section{Introduction}

Electric charge in liquids is presently measured mostly through global, non-resolutive approaches like impedance spectroscopy, cyclic voltammetry or flow electrification [1-4]. The resolutive Kerr effect technique [5,6] is limited in sensitivity when applied to a dielectric liquid by low values of the Kerr constant and by the sensitivity of the camera used for the experiment, and can only be used in transparent liquids. Thus, sensitivity and spatial resolution available through existing methods for quantifying and localizing electric charges in liquids are significantly restricted. It is to note that the theoretical distribution of the electrical double layer, suggested by Stern in 1924 [7] and fully admitted since, has never been yet confirmed experimentally, mainly due to the lack of sensitivity and resolution of the available methods. Thus, thorough study of the formation and the evolution of charged areas in liquids and at liquid/solid interfaces are still requiring new measurement techniques. In this context, the application of stimuli methods [8,9] to dielectric liquids [10-20], particularly thermal methods like the thermal step method (TSM) [21-25], is receiving increasing attention.

Indeed, extensive studies of electric charge effects in solid dielectrics have been made possible by the development of non destructive methods for in such materials $[8,9]$. Amongst them, thermal methods are the most sensitive [26] and lie on the diffusion of heat, which generates a slight local disequilibrium of the volume space charge $\rho(V)$ and a slight local variation of the permittivity $\varepsilon$, leading to a variation of the induced charges at electrodes $Q_{1}$ and $Q_{2}\left(Q_{1}+Q_{2}+Q_{i}=0\right.$ due to the charge conservation law, with $\left.Q_{i}=\iiint_{V} \rho(V) d V\right)$. The result is an electrical response, which, for a short-circuited sample submitted to inhomogeneous variation of temperature across its thickness with time $t$, is a transient current:

$$
I(t)=-\partial Q_{1} / \partial t=\partial Q_{2} / \partial t
$$


In the case of a flat homogeneous solid sample submitted to a heat pulse or temperature step (Figure 1), this signal reads [21-24]:

$$
I(t)=-\alpha C \int_{0}^{d} E(x) \frac{\partial \Delta T(x, t)}{\partial t} d x
$$

where $C$ is the capacitance of the sample, $\alpha=\alpha_{x}-\alpha_{\varepsilon}=(1 / x) \times \mathrm{d} x / \mathrm{d} T-(1 / \varepsilon) \times \mathrm{d} \varepsilon / \mathrm{d} T$ is a coefficient of relative variation with temperature $T$ of the abscissae $x$ and of the permittivity $\varepsilon, d$ is the thickness of the sample, $E(x)$ is the electric field distribution across the sample in the thickness direction (which is a depth coordinate: $x \in[0, d]$ ) and $\partial \Delta T(x, t) / \partial t$ is the law of variation of the temperature with time at the abscissa $x$.

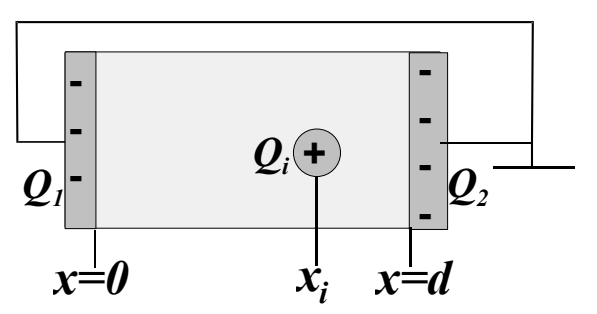

(a)

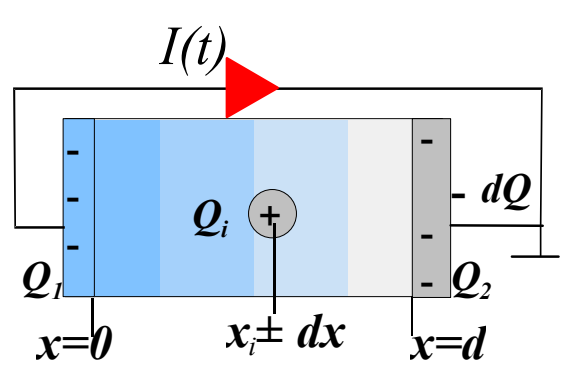

(b)

Figure 1: Principle of a thermal stimulus method (example for a sample in short-circuit). The presence of a space charge $Q_{i}$ induces influence charges $Q_{1}$ and $Q_{2}$ at electrodes $(a)$. When the sample equilibrium is perturbed by a thermal stimulus applied to the left electrode, charge variation at electrodes $\left(Q_{2}-d Q\right.$ and $Q_{1}+d Q$, respectively) occurs to restore it. $T h u s$, the transport of charge $d Q$ from an electrode toward the other through the external circuit results in a transient current response $I(t)=-d Q / d t$.

In coaxial geometry (Figure 2), by neglecting the axial dimension, the transient current signal reads:

$$
I(t)=-\alpha C \int_{R_{\text {int }}}^{R_{\text {ext }}} E(r) \frac{\partial \Delta T(r, t)}{\partial t} d r
$$

where $R_{\text {int }}$ and $R_{\text {ext }}$ are the inner and the outer radii of the coaxial insulation, $E(r)$ is the radial electric field distribution across the coaxial sample $\left(r \in\left[R_{\text {int }}, R_{\text {ext }}\right]\right)$ and $\partial \Delta T(r, t) / \partial t$ is the law of variation of the temperature with time at the radius $r$. 


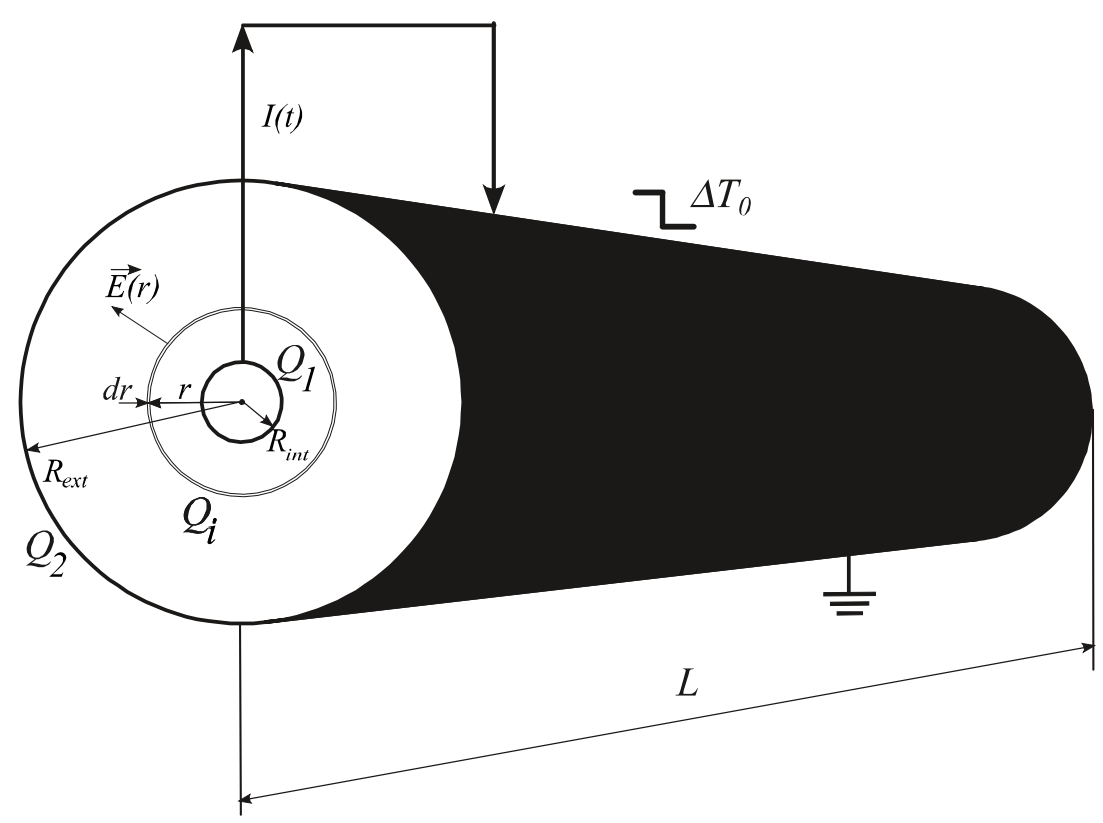

Figure 2: Principle of a thermal stimulus method in a coaxial geometry.

The electric field and charge distributions across $x$ or $r$ can be determined by solving the above equations as described in [21,25,27], provided that these distributions vary much slowly than the measurement time, which is usually the case for solids.

If this principle is à priori applicable to a sample of dielectric liquid, one must take into account that, in addition to thermal conduction, thermal convection, which does not exist in a solid sample, may occur in a liquid one. Charge transport through thermal convection may then affect the charge and field distributions in the liquid during the measurement. This may lead to errors in processing the measured signals, as the condition of slow variation of the field distribution with respect to the measurement time, required for solving equations (1) and (2), could be no longer fulfilled. 
In this paper, we approach, through numerical analysis, the effects of thermal convection on the electrical responses of liquid insulating samples submitted to a thermal stimulus, and derive criteria to assess and limit them.

\section{Calculation model}

\subsection{Calculation domain}

The studied configuration is that of a coaxial liquid sample from Figure 3. The electrical double layer [7], which appears when any liquid is set into contact with a solid wall, is used in this work as a study support. Thus, a charge distribution comprising the two diffuse charge layers set up near each electrode [7,28] is considered across the sample (Figure 3). As in pure dielectric liquids like hydrocarbons the Debye length $\delta_{0}$ is of the order of $100-150 \mu \mathrm{m}$ at the most [29,30], which gives a maximum total length of the charged area $2 \times 5 \times \delta_{0}=1.5 \mathrm{~mm}$, a thickness of $3 \mathrm{~mm}$ was chosen for the liquid sample (3) to exclude interpenetration of the two charge zones. The other dimensions and the sample geometry were set to obtain a capacitance higher than $100 \mathrm{pF}$ (of the order of those used with the TSM in solid samples of similar thicknesses) and to be as close as possible to a practically feasible and reliable measurement cell and experimental setup.

The $1 \mathrm{~mm}$-thick electrodes (domains (2) and (4)) are considered as of stainless steel, with density $\rho_{m}=7780 \mathrm{~kg} / \mathrm{m}^{3}$, specific heat $C_{p}=540 \mathrm{~J} \cdot \mathrm{kg}^{-1} \cdot \mathrm{K}^{-1}$ and thermal conductivity $\lambda=19.83 \mathrm{~W} \cdot \mathrm{m}^{-1} \cdot \mathrm{K}^{-1}$. The measurement cell is assumed as initially at $T_{0}=20^{\circ} \mathrm{C}$. The thermal excitation is considered as applied by a flow of ethanol at a temperature $T_{0}+\Delta T_{0}\left(\Delta T_{0} \in\left[-20^{\circ} \mathrm{C}\right.\right.$, $\left.+20^{\circ} \mathrm{C}\right]$ ), circulating through the inner chamber (domain $(1)$ ) at a speed of $5 \mathrm{~m} / \mathrm{s}$. The considered properties of ethanol are, at room temperature: density $\rho_{m}=808 \mathrm{~kg} / \mathrm{m}^{3}$, specific heat $C_{p}=2400 \mathrm{~J} \cdot \mathrm{kg}^{-1} \cdot \mathrm{K}^{-1}$, dynamic viscosity $\mu_{d y n}=1.2 \times 10^{-3} \mathrm{~Pa} \cdot \mathrm{s}$ and thermal conductivity $\lambda=0.168 \mathrm{~W} \cdot \mathrm{m}^{-1} \cdot \mathrm{K}^{-1}$. Their variations with temperature are: 


$$
\begin{gathered}
\rho_{m}(T)=475.01+4.71 \times T-0.0175 \times T^{2}+1.83 \cdot 10^{-5} \times T^{3} \\
C_{p}(T)=4012.27-18.71 \times T+0.0451 \times T^{2} \\
\mu_{d y n}(T)=0.07414-6.170 \cdot 10^{-4} \times T+1.74 \cdot 10^{-6} \times T^{2}-1.66 \cdot 10^{-9} \times T^{3} \\
\lambda(T)=0.2190-1.221 \cdot 10^{-4} \times T-1.79 \cdot 10^{-7} \times T^{2}
\end{gathered}
$$

The external chamber (domain (5)) is considered as air-filled to act as a thermal insulator.

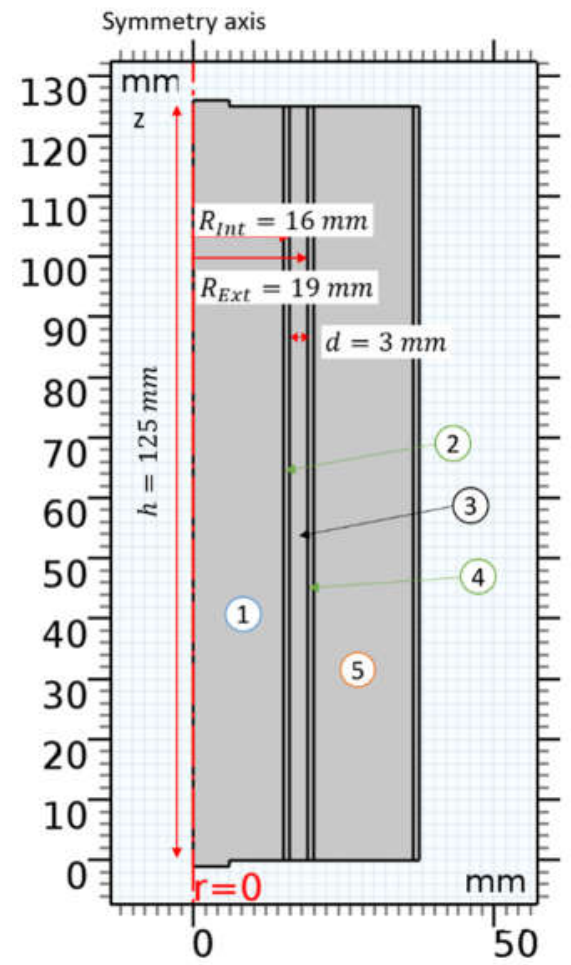

(a)

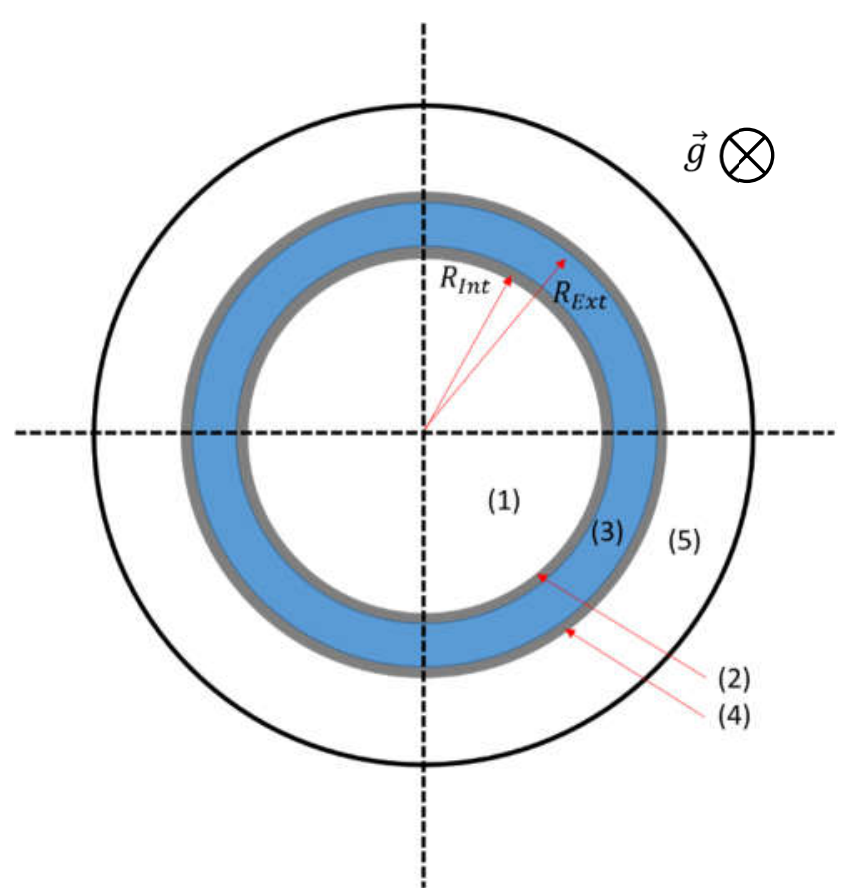

(b)

Figure 3:Axial (a) and top (b) views of the cell and computation domains considered for numerical calculations of transient currents corresponding to the application of a thermal stimulus to a dielectric liquid. (1): Chamber used for applying the thermal stimulus through a flowing cold or warm liquid, (2) and (4): Stainless steel walls (electrodes), (3): Studied dielectric liquid (sample), (5): Air-filled (thermally insulating) chamber. $\vec{g}$ stands for the gravity acceleration. 


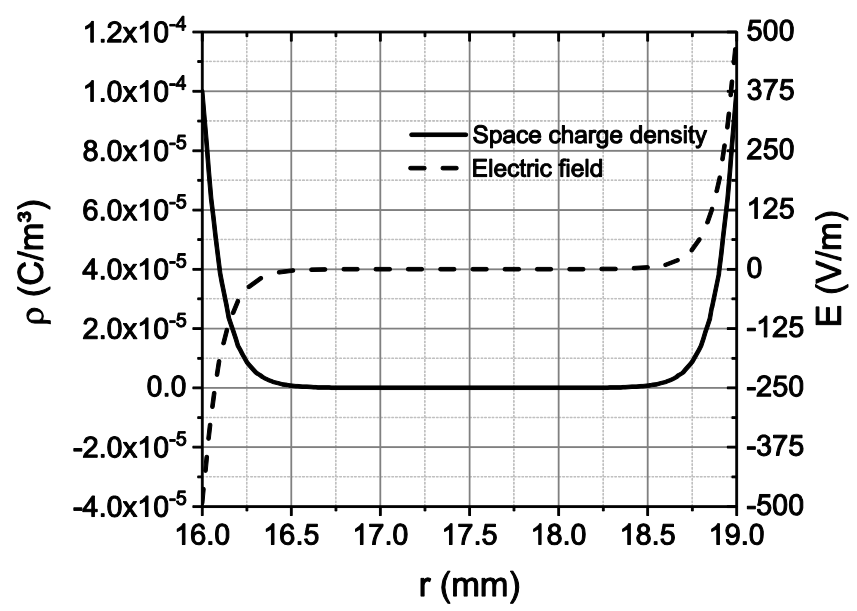

Figure 4: Diffuse-layer-like assumed space charge density and electric field distributions across the insulating liquid.

Because thermal convection is related to dynamic viscosity, two generic liquids with different viscosities were considered (domain (3)). The first is a low-viscosity one, with the properties of cyclohexane, i.e.: dynamic viscosity $\mu_{d y n}=0.980 \times 10^{-3} \mathrm{~Pa} \cdot \mathrm{s}$, thermal diffusivity $D_{T}=1.38 \times 10^{-7} \mathrm{~m}^{2} / \mathrm{s}$ and density:

$$
\rho_{m}(T)=84.1595 \times \frac{0.8908}{0.27396^{1+\left(1-\frac{T}{553.58}\right)^{0.2851}}}
$$

The second one is a higher viscosity liquid, representative of transformer mineral oils, with $\mu_{d y n}=17 \times 10^{-3} \mathrm{~Pa} \cdot \mathrm{s}, \rho_{m}=850 \mathrm{~kg} / \mathrm{m}^{3}$ at $20^{\circ} \mathrm{C}, D_{T}=0.93 \mathrm{~m}^{2} / \mathrm{s}$ and a volumetric coefficient of thermal expansion $\beta=7 \times 10^{-4} \mathrm{~K}^{-1}$.

\subsection{Solved equations}

The space charge and electric field distributions from Figure 4, representing typical profiles in insulating liquids at the liquid/wall interface in the hypothesis of weak charge densities [28], were considered for both liquids. The sample geometry $\left(R_{\text {int }}\right.$ and $R_{\text {ext }}>>\left(R_{\text {ext }}{ }^{-}\right.$ $\left.R_{\text {int }}\right)$ ) allows considering charge profiles decreasing exponentially from the walls toward the bulk [31]: 


$$
\begin{aligned}
& \rho(r)=\rho_{w d} \times \exp \left(-\frac{r-R_{\text {int }}}{\delta_{0}}\right) \text { for } r \in\left[R_{\text {int }} ;\left(R_{\text {ext }}+R_{\text {int }}\right) / 2\right) \\
& \rho(r)=\rho_{w d} \times \exp \left(\frac{r-R_{\text {ext }}}{\delta_{0}}\right) \text { for } r \in\left[\left(R_{\text {ext }}+R_{\text {int }}\right) / 2 ; R_{\text {ext }}\right]
\end{aligned}
$$

where $\rho_{w d}=10^{-4} \mathrm{C} / \mathrm{m}^{3}$ is the space charge density at the wall. This profile has been set as initial distribution for the computation.

The electric field $E$ and the electric potential $U$ across the sample are calculated by using the Poisson and the electrostatic potential equations:

$$
\begin{gathered}
\vec{\nabla} \cdot\left(\varepsilon_{0} \varepsilon_{r} \vec{E}\right)=\rho \\
\vec{E}=-\vec{\nabla} U
\end{gathered}
$$

The thermal stimulus is assumed as applied through the cold or warm fluid circulating in the inner cavity (1) from top to bottom. The temperature variation due to heat diffusion through the stainless steel walls (2) and (4) are computed with the heat equation, i.e. equation (12) where $v=0$. Heat diffusion and convection within the sample (domain (3)) are computed by linking the heat equation and the Navier-Stokes equations in incompressible flow with the Boussinesq approximation [32]:

$$
\begin{gathered}
\rho_{m} C_{p} \frac{\partial T}{\partial t}+\rho_{m} C_{p} \vec{v} \cdot \vec{\nabla} T-\lambda \overrightarrow{\nabla^{2}} T=\dot{q}_{v} \\
\rho_{m}\left(T_{0}\right)\left[\frac{\partial \vec{v}}{\partial t}+(\vec{v} \cdot \vec{\nabla}) \vec{v}\right] \\
=-\vec{\nabla} p+\vec{\nabla} \cdot\left\{\mu_{d y n}\left[\vec{\nabla} \vec{v}+(\vec{\nabla} \vec{v})^{T}-\frac{2}{3}(\vec{\nabla} \cdot \vec{v}) \mathrm{I}\right]\right\}+\rho_{m}\left(T_{0}\right) \beta\left(T-T_{0}\right) \vec{g} \\
\vec{\nabla} \cdot(\vec{v})=0
\end{gathered}
$$


with $C_{p}$ specific heat, $\vec{v}$ velocity, $\lambda$ thermal conductivity, $\dot{q}_{v}$ heat source, $T_{0}$ initial temperature and $p$ pressure. The electro-hydrodynamic forces were neglected due to the low values of the electric field from Figure 4 (this hypothesis was also verified by preliminary simulations).

The charge $\rho$ is assumed as transported by the thermal convection flow through a transport equation where diffusion is neglected with respect to convection, and also the charge creation, which is far slower than the considered measurement time:

$$
\frac{\partial \rho}{\partial t}+\vec{\nabla}(\vec{v} \rho)=0
$$

To considerably reduce simulation times by excluding the mechanic physics, $\alpha=-\alpha_{\varepsilon}=10^{-4} \mathrm{~K}^{-1}$ (of the order of values measured experimentally on cyclohexane and on a widely used transformer oil) is considered for both liquids, i.e. in the domain (3) the following law of variation of the relative permittivity with temperature is imposed:

$$
\varepsilon_{r}(x, t)=\varepsilon_{r}\left(T_{0}\right) \times\left[1+\alpha_{\varepsilon}\left(T(x, t)-T_{0}\right)\right]
$$

Two situations were analyzed: without and with considering thermal convection within the liquid sample. The first case, identical to what occurs in a solid dielectric in terms of thermal transfer, is used as reference for the sole effect of thermal conduction on the electric response. The second one is the result of both thermal transfer mechanisms (conduction and convection) and allows assessing the impact of thermal convection on the signal. In both cases, the signals are computed by integrating numerically the displacement current density $\vec{J}_{D}=$ $\partial \vec{D} / \partial t=\partial(\varepsilon \vec{E}) / \partial t$ on the surface $S$ of one of the electrodes:

$$
I(t)=\int_{S} \vec{J}_{D} d \vec{S}
$$


In the domain (1) (corresponding to the flow of ethanol), the turbulent flow, thermal conduction and thermal convection coupled to turbulent flow physics are considered. The ethanol flow is considered as entering through the upper side of the domain and exiting through the lower end, through an inlet with a radius $r_{\text {inlet }}=6 \mathrm{~mm}$. The mesh in domain (1) is optimized for fluid dynamics - it is highly refined near the interfaces, where the velocity gradients are significant (Figure 5). The fluid velocity input condition is set as a normal velocity of norm $v_{n}=5 \mathrm{~m} / \mathrm{s}$.

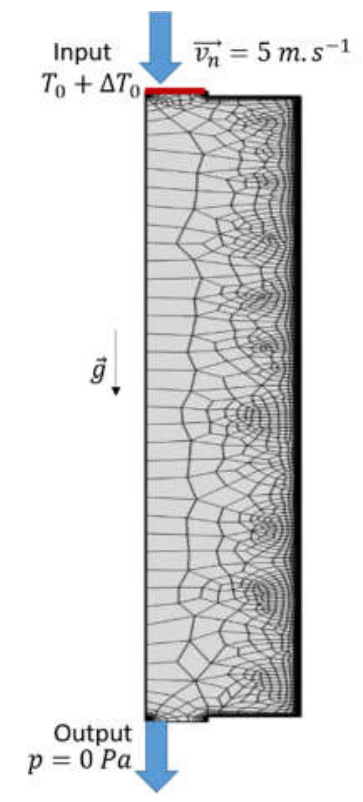

Figure 5: Mesh in the domain (1) (flowing heating fluid). $g$ stands for gravity acceleration and $v_{n}$ for the fluid velocity at the domain input.

It is considered that a flow of ethanol at $T_{0}$ circulates in the domain (1) before the simulated experiment (stationary flow regime at $T_{0}$ with the abovementioned velocity at the top of domain (1)). To simulate the application of a thermal stimulus of amplitude $\Delta T_{0}$ with the aid of the ethanol flow, it is assumed that at $t=0$ the temperature of the ethanol entering the domain (1) switches to $T_{0}+\Delta T_{0}$. Thus, to initialize the velocity profile, a stationary calculation of the turbulent flow at the initial temperature $T_{0}$ is carried out and then a temporal step, where the thermal step is applied, is launched. 
The velocity profiles in the domain (1), calculated for ethanol temperatures of $0^{\circ} \mathrm{C}, 20^{\circ} \mathrm{C}$ and $40^{\circ} \mathrm{C}$ (by taking into account the variation of the ethanol properties with temperature), are represented in Figure 6 . The differences in velocity values are less than $1 \%$. For this reason and with the goal of decreasing the calculation time, the fluid movement in the domain (1) determined in the stationary stage is not computed any more in the temporal step; the velocity profile calculated in the stationary stage is kept and set as an initial condition.

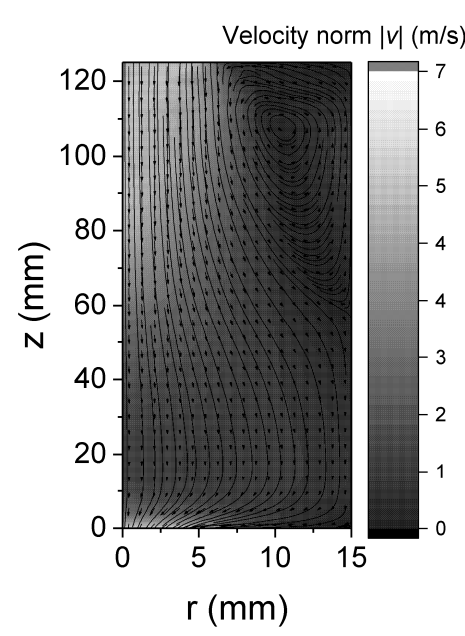

(a)

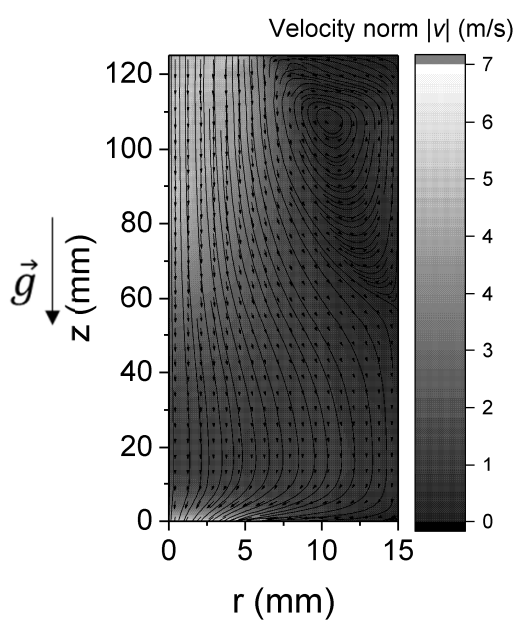

(b)

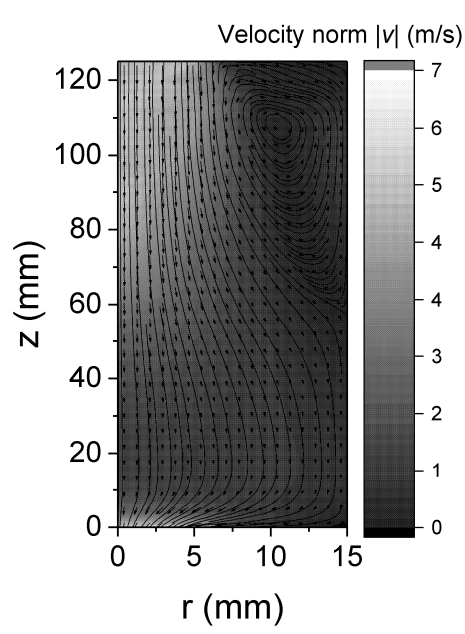

(c)

Figure 6: Profiles of the velocity norm $|\vec{v}|$ of the heating fluid (domain (1)) in stationary regime for a temperature of the fluid of $0^{\circ} \mathrm{C}, 20^{\circ} \mathrm{C}$ and $40^{\circ} \mathrm{C}$

In the stainless steel walls (domains (2) and (4)), only the heat equation is solved. The mesh is here conditioned by the domains of the heating fluid (1) and of the sample (3), and require few meshing elements in the direction of the heat flux to make the calculations converge.

In the sample domain (3), the thermal conduction, electrostatics, laminar flow and coupled charge transport equations are considered. At the boundaries of domain (3), the mesh is highly refined (Figure 7), with a first mesh of $1 \mu \mathrm{m}$ in width and then a growth rate of 1.2 for the first $350 \mu \mathrm{m}$ close to the interfaces in the $r$-direction. For the rest of the bulk, the mesh elements are $50 \mu \mathrm{m}$-wide in the $r$-direction. In the $z$-direction (height), the meshes are $500 \mu \mathrm{m}$ - 
high, except near the fluid input and output, where the mesh is much more refined, with a first element of $5 \mu \mathrm{m}$ in height and a growth rate of 1.2 for 15 mesh elements.

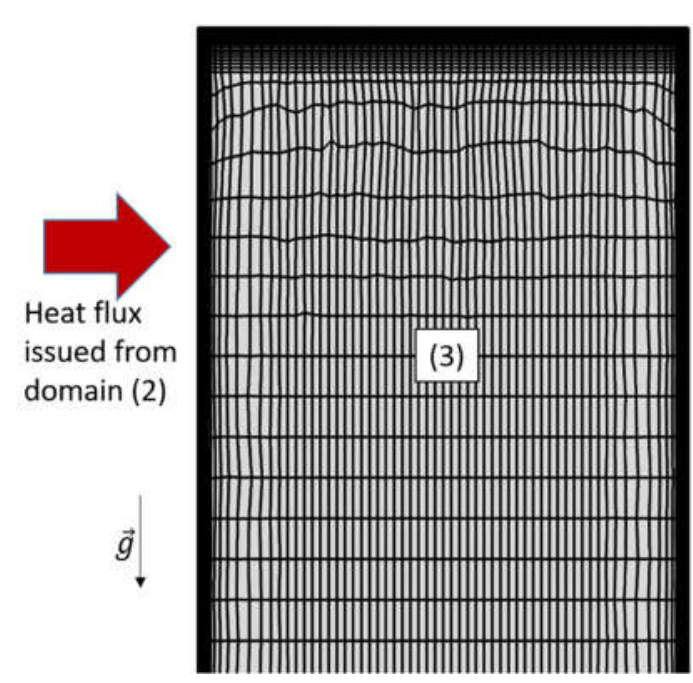

Figure 7: Mesh of the liquid sample (domain (3)) for a height $z$ comprised between $121 \mathrm{~mm}$ and $125 \mathrm{~mm}$

Domain (5) is considered as a chamber filled with air at the temperature $T_{0}$, to act as a thermal screen. In domains (2) and (4), only the heat equation is solved. The condition on the external boundary is the following: if the heat flux "enters" the domain, the external temperature is equal to $T_{0}\left(T=T_{0}\right)$, otherwise the outgoing heat flux $q_{h}$ is nil $\left(-\vec{n} \cdot \overrightarrow{q_{h}}=0\right.$, with $\vec{n}$ the unit vector normal to the wall). A relaxed mesh is used here, as the heat flux has barely the time to reach this domain, which mainly serves as a limit condition for the rest of the geometry.

\section{Results and discussion}

Figure 8 shows transient currents (thermal step (TS) signals) computed with the low and high viscosity liquids without taking into account the thermal convection movements. For each liquid, the currents are strictly proportional to the amplitude of the temperature stimulus $\Delta T_{0}$. This is in accordance with the analytical expression of the current (2) when a thermal stimulus of the form $\Delta T(r, t)=\Delta T_{0} \times f(t)$ is applied, as in the case of a solid sample, where only thermal conduction occurs. The small differences between the signals of the two liquids are due to their difference in thermal diffusivity. 


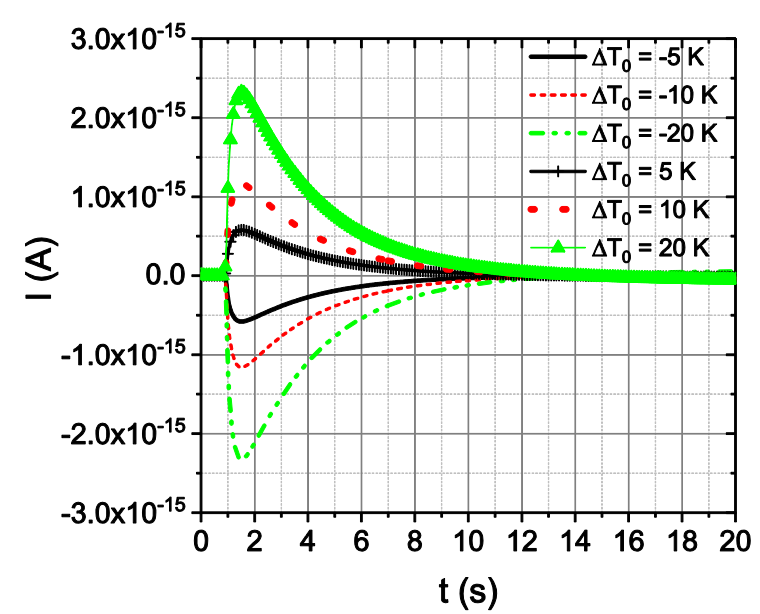

(a)

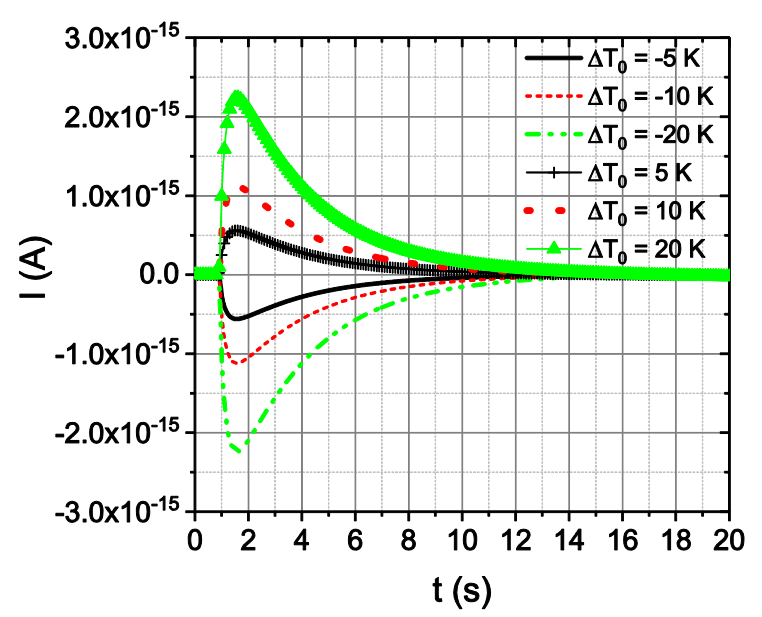

(b)

Figure 8: Thermal step currents computed in the low viscosity (a) and viscous liquids (b) when stimuli of different amplitudes $\Delta T_{0}$ are applied, without considering thermal convection movements

TS currents calculated when considering both thermal transfer by conduction and the thermal convection motions are shown in Figure 9. In contrast to Figure 8a), where the signs of the currents revert when the sign of the thermal stimulus is reverted, the currents corresponding to the low-viscosity liquid ( Figure 9a) keep the same sign, whatever the thermal stimulus. This provides an interesting indication for detecting a major effect of charge displacement by convection in a measured signal.

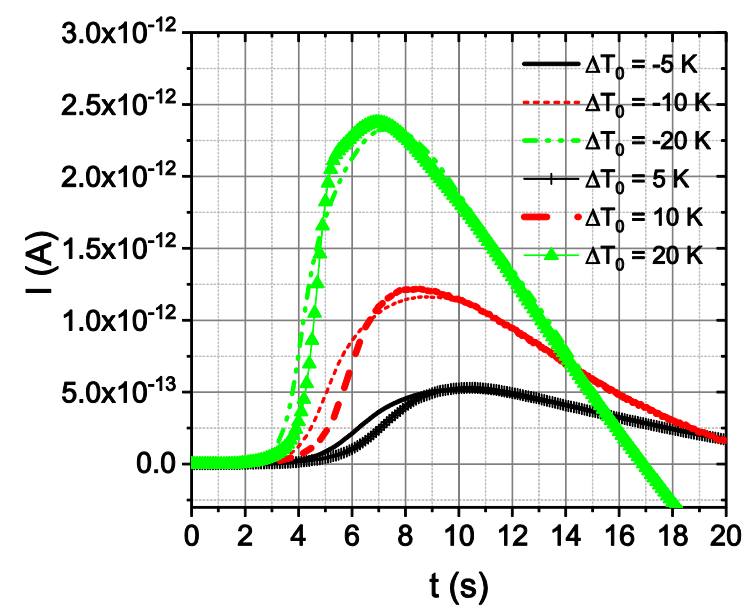

(a)

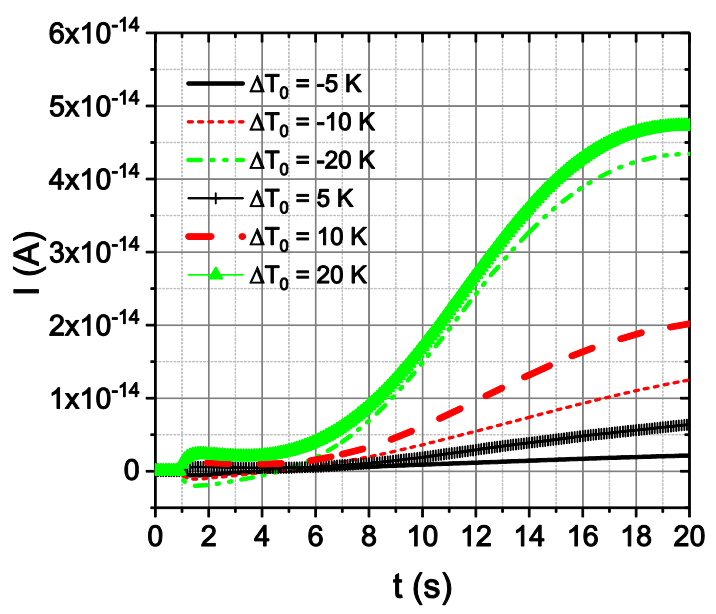

(b)

Figure 9: Thermal step currents computed in the low viscosity (a) and viscous liquids (b) when stimuli of different amplitudes $\Delta T_{0}$ are applied, with the thermal convection movements taken into account. 


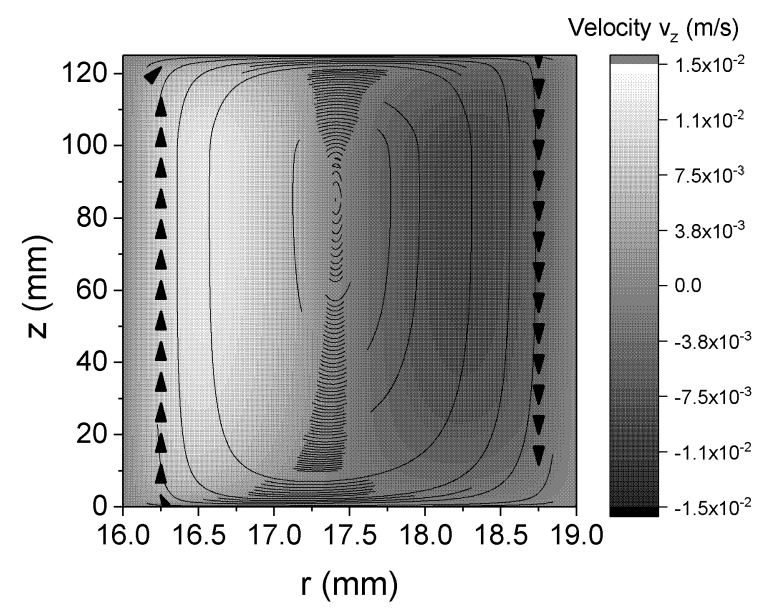

(a)

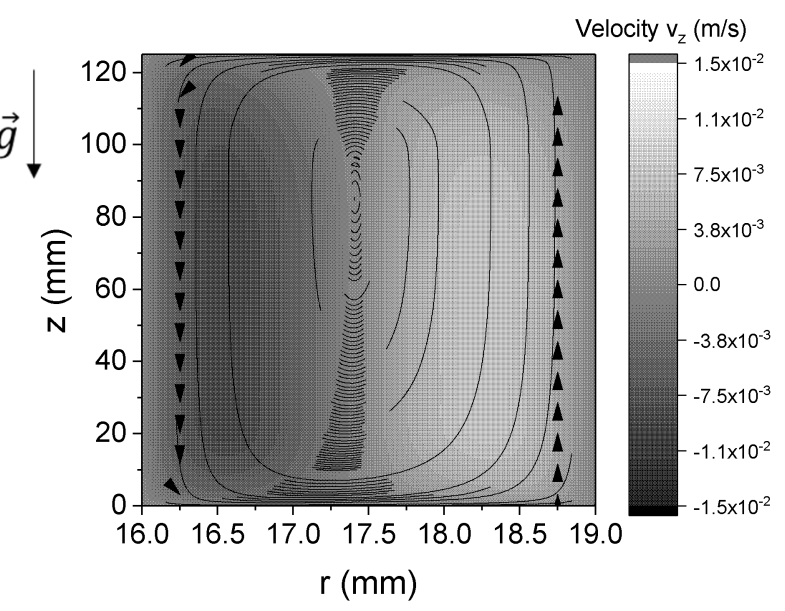

(b)

Figure 10: Profiles of velocity along the z-axis due to convection movements in the low-viscosity liquid (domain (3)) when a thermal stimulus of $+20 \mathrm{~K}(\mathrm{a})$ and $-20 \mathrm{~K}(\mathrm{~b})$ is applied, at $t=8 \mathrm{~s}$ after the application of the stimuli.

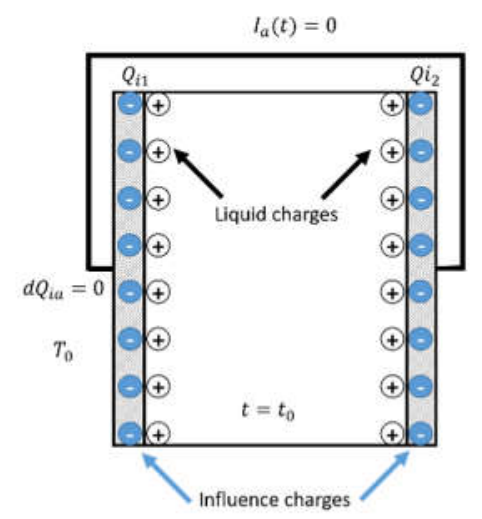

(a)

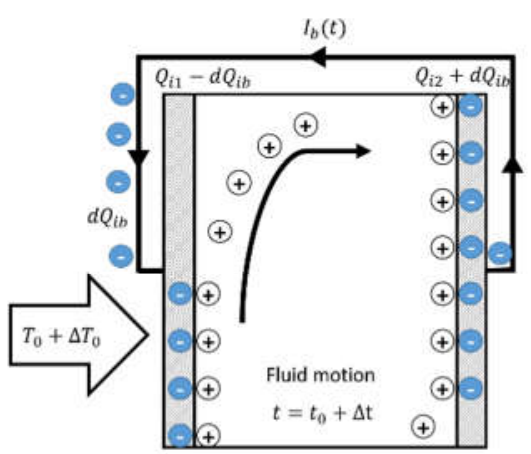

(b)

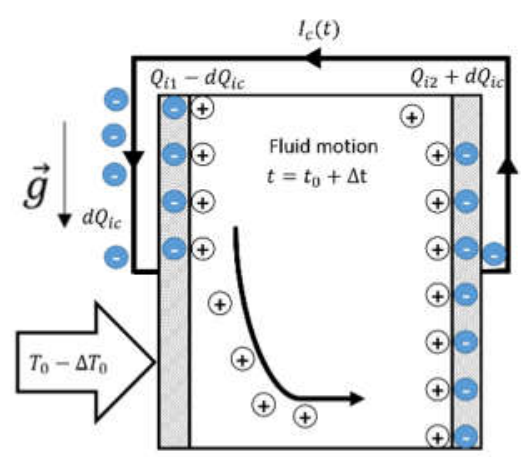

(c)

Figure 11: Electric charges in the liquid before applying the thermal stimulus (a) and displacement of these charges by the thermal convection flow during the application of a positive (b) or negative (b) stimulus. The sense of the liquid movement in the convection cell reverts when the thermal stimulus is reverted, but the charges from the liquid are always

convected from left to right (clockwise for a positive thermal stimulus and counterclockwise for a negative one). Consequently, the redistribution of influence charges occurs in both cases in the same sense, and the TS currents appearing in the external circuit $\left(I_{b}(t)\right.$ and $\left.I_{c}(t)\right)$ have the same sense.

To interpret the fact that, despite the reversal of the thermal stimulus sign, the thermal stimulus current does not revert when the charge convection by the liquid flow is taken into account, we plotted in Figure 10 the velocity lines of the convection movements along the $z$ axis in the cases of a positive and a negative thermal step with $\left|\Delta T_{0}\right|=20 \mathrm{~K}$. The charges from the interface layers (i.e., placed near $r=16 \mathrm{~mm}$ and $r=19 \mathrm{~mm}$ ) are displaced by the liquid movements following the convection lines, clockwise or counterclockwise, with respect to the sign of the stimulus. However, even if the sense of the convection movements of the fluid is 
reverted when the sign of the thermal stimulus reverts, these convection movements always transport the charges from the charged layers of the fluid toward the opposite electrode. The fluid movements provoked by thermal convection are clockwise or counterclockwise, with respect to the sign of the stimulus, but the net displacement of the charge in the $r$-direction is always in the same sense, as illustrated in Figure 11. Consequently, the transport of influence charges from an electrode to the other via the outer circuit is always made in the same sense, so the thermal step current induced by the thermally convected charges is always of the same sign.

On the other hand, when comparing Figures 8a) and 9a), it comes out that the part of the signal due to the thermal conduction (which will be called so far "conductive component") is so small with respect to the "thermo-convective" component that it becomes invisible when the thermal convection is included in the simulation. This clearly indicates that the signals from Figure 9a) are due in very large part to thermal convection effects.

When thermo-convection effects are considered, the rising dynamics of the signals is more important for a negative thermal stimulus. This is related to the velocity of the liquid near the interface, which depends on the variation of the specific density with temperature (equation (8)). Thus, gravity amplifies the descendant flow velocity at the interface when a negative stimulus is applied, as the specific density increases when temperature decreases. Once a convection cell is established (i.e., after $10 \mathrm{~s}$ ), the currents issued from opposite temperature steps of same amplitude overlap, showing that once the convection flow is set, the effects of gravity on a low viscosity liquid are negligible.

The dynamics of the response of the more viscous liquid ( Figure $9 \mathrm{~b}$ ) is slower, as a result of a lower flow velocity due to its higher viscosity. It takes longer to set this liquid in motion, allowing to observe a supplementary current peak for $t<2 \mathrm{~s}$. This peak, which appears at the same moment as the peaks from Fig. 8b), and which behaves in the same way as them (sign 
inversion when the sign of the stimulus is reverted, proportionality between the amplitude of the peak and that of the stimulus), is due to heat diffusion by conduction, as in a solid. A classical "thermo-conductive" behavior (solid-like) is thus observed at least at the beginning when viscosity increases.

After several seconds, the convection flow establishes and the contribution of thermal convection becomes preponderant, as for the low viscosity liquid. However, the values of the current are lower for the more viscous liquid, due to the velocity of the flow, which decreases when viscosity increases. The rising slopes are almost identical for positive and negative steps (the delay being mainly due to the contribution of the "conductive" component at the beginning of the signal) and the sign of the "convective" component is also the same, whatever the sign of the stimulus. To illustrate this, Figure 12 shows currents computed by considering:

i) the variation of permittivity with temperature, but without charge transport by the convection flow (no convection and $\alpha=10^{-4} \mathrm{~K}^{-1}$, curve i);

ii) charge transport by the thermal convection flow, but no permittivity variation (only convection and $\alpha=0$, curve ii);

iii) charge transport by thermal convection and permittivity variation with temperature (convection and $\alpha=10^{-4} \mathrm{~K}^{-1}$, curve iii).

The sum of the curves (i) and (ii) is also plotted, and it perfectly superposes to (iii).

Figure 12 underlines that below $4 \mathrm{~s}$, the "convective" component is very small, and the TS current is reduced to its "conductive" component. As the convection cell is establishing, the "convective" component increases rapidly, up to become highly major. The "conductive" component changes sign with the temperature step, but its contribution to the overall signal is low because its value is highly supplanted by the "convective" component, except at the beginning of the heat transfer process. 


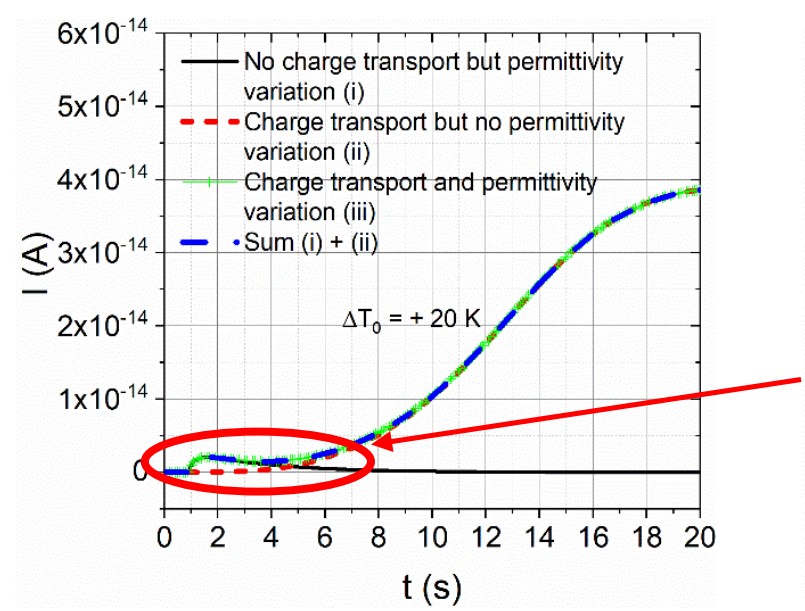

(a)

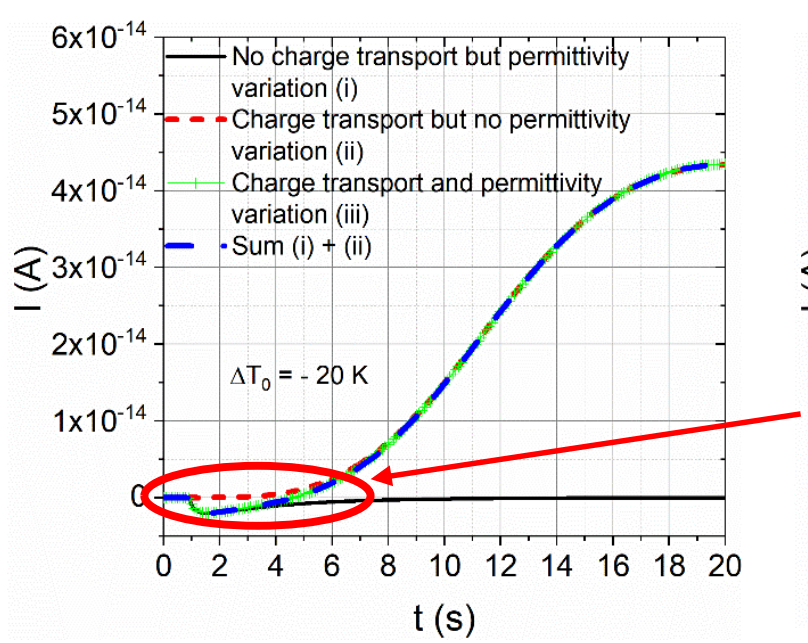

(c)

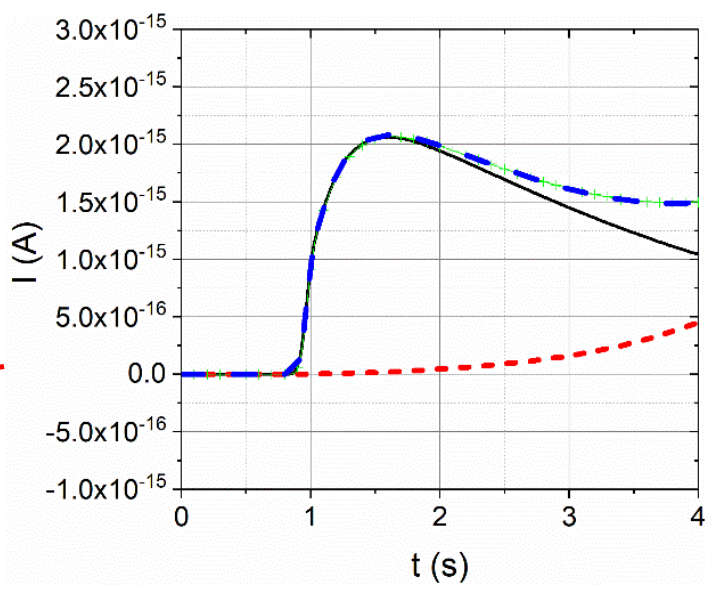

(b)

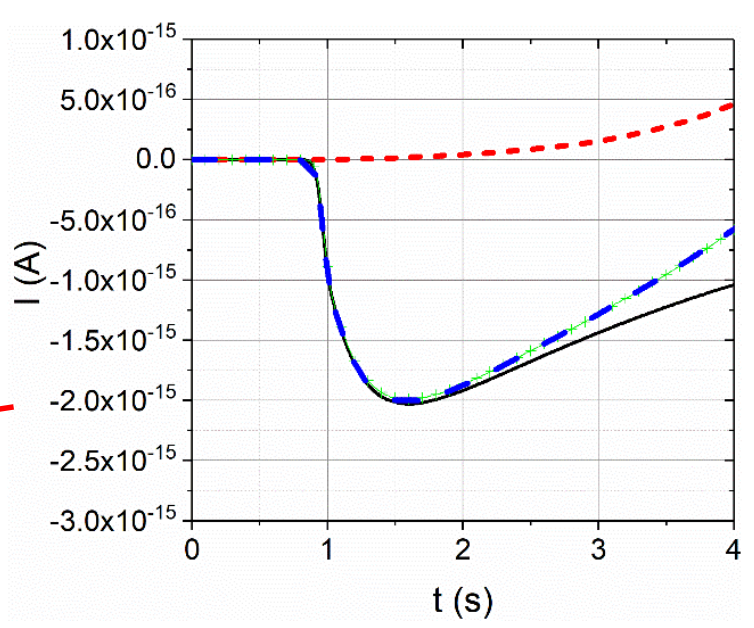

(d)

Figure 12: Comparison of "conductive", "convective" and "total" currents computed for the viscous liquid under temperature steps of $20 \mathrm{~K}(\mathrm{a})$ and $-20 \mathrm{~K}(\mathrm{c})$ and zoom at the beginning of the signals $(b)$ and (d).

These results allow drawing several useful conclusions for interpreting experimental data and identifying the presence of convection effects. They show that measuring signals of the same sign and of close amplitudes when the sign of the stimulus reverts is characteristic of a major contribution of thermally convected charges. Likewise, a stall followed by a strong increase of the current (case of the viscous fluid) may witness a first stage where the entrainment 
of the charges by the fluid is negligible, followed by a second stage where the signal is mainly due to charge movements by thermo-convection.

It comes out from the above results that, for avoiding errors in determining electric field distributions, the validity of equations (2) or (3) must be checked by verifying that the thermoconvective movements are absent, or at least that their contribution is much smaller than that given by the variation of permittivity and abscissae with temperature. If this is certainly the case for very viscous liquids, it could not be the case for a large variety of dielectric liquids with respect to used experimental set-ups.

Thermal convection can be substantially reduced with appropriate set-ups. For instance, a measurement cell with the same capacitance and sample thickness as that from Figure 3, but in a flat horizontal geometry, and where a positive thermal stimulus is applied downward, is shown in Figure 13a). The calculated liquid velocity in the sample due to thermal convection is here less than $2 \times 10^{-4} \mathrm{~mm} / \mathrm{s}$ (which is the numerical noise threshold) with respect to $15 \mathrm{~mm} / \mathrm{s}$ in the configuration from Figure 3 (as shown by the data from Figure 10). Consequently, in the flat cell the thermal convection is practically zero, and the currents computed with and without the "convective" term superpose perfectly (Figure 13b).

Setting up a flat cell with such dimensions, simulated here for comparison reasons, is apparently easy, but such a structure would be hardly reliable from a mechanical point a view, considered its large dimensions and length over thickness ratio. However, cells with smaller dimensions and thicknesses can be manufactured with present technologies, to fully allow measuring and studying charge distributions in dielectric liquids and at their interfaces without interference of thermal convection phenomena. To reach this aim, thermo-convective responses should be carefully assessed in any experimental set-up with respect to its geometry and 
dimensions, by taking into account the form, the intensity and the duration of the used thermal stimulus.

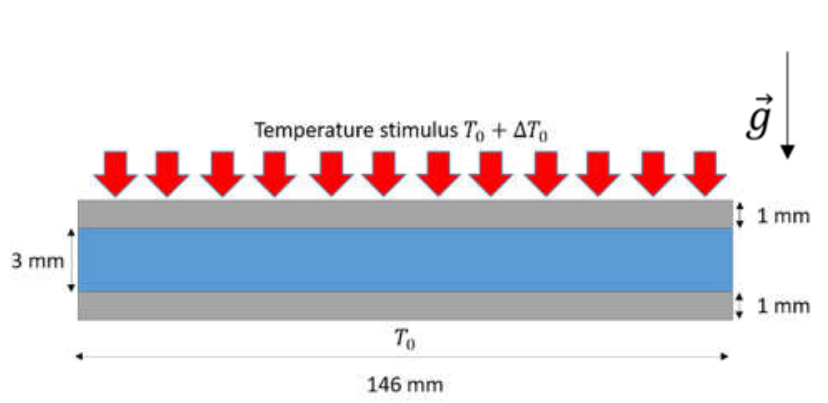

(a)

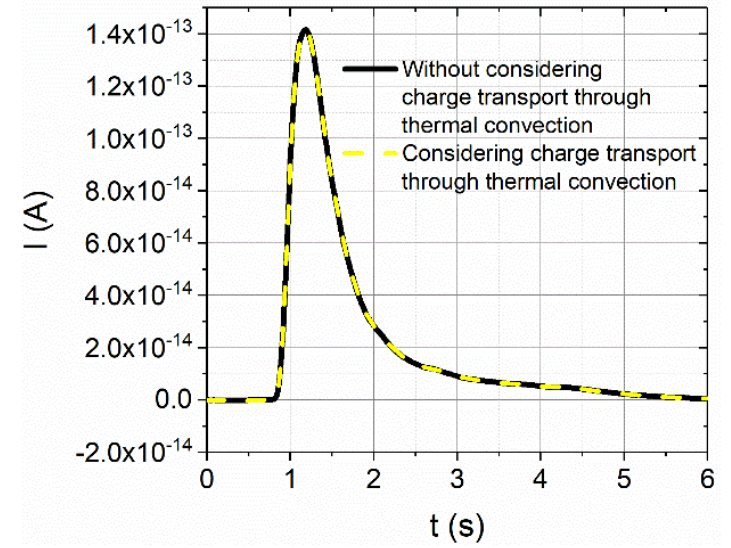

(b)

Figure 13: Measurement cell configuration allowing to substantially reduce thermal convection effects (a) and signals computed in the cell with and without considering charge transport by thermal convection effects (b) in the low viscosity liquid after the application of a thermal stimulus $\Delta T_{0}=20 \mathrm{~K}$

\section{Conclusions}

A thermal stimulus can be used to assess the distribution of electrical charges within a liquid and in the electrical double layer. The idea is to provoke, by this excitation, a reversible electrostatic imbalance without generating a movement of the fluid by thermo-convective effect.

The numerical simulations showed that, for a cylindrical cell with a vertical axis and a thermal excitation produced parallel to the axis through a heating fluid pushed downward, thermo convective rollers are generated in the studied liquid, in spite of small temperature steps. This phenomenon is all the less marked as the viscosity of the liquid is important.

The electrical response then contains two pieces of information: one related to the response of the liquid charges in their immobile state, the other related to the transport of the liquid charges by the convection phenomenon. The analysis of the results shows that, in terms 
of amplitude, the thermo-convective effects may prevail, but they develop at a slower dynamics than the "static" effects of the charge in the liquid. The measured signal would be mainly due in a first step (during the 2 to 3 seconds sufficient for the diffusion of the thermal wave throughout the liquid sample) to the "static" effect of the charge, then in a second step the thermo-convective effects would dominate.

On the other hand, the convective effects are independent of the sign of the temperature stimulus (positive or negative), contrary to the effects of a static liquid charge, which constitutes an important discriminating parameter in the analysis of the results. Finally, it is shown that the use of a cell of plane geometry, where a positive thermal stimulus is applied downwards, would make it possible to avoid the thermo-convective effects when measuring the electric charges in the liquid.

\section{Acknowledgment}

This work was supported by the French government program "Investissements d'Avenir" (LABEX INTERACTIFS, reference ANR-11-LABX-0017-01). 


\section{References}

[1] A.J. Bard, L.R. Faulkner, Electrochemical Methods: Fundamentals and Applications, New York: Wiley, 2001, 2nd ed., Russian Journal of Electrochemistry. 38 (2002) 1364-1365. https://doi.org/10.1023/A:1021637209564.

[2] M.E. Orazem, B. Tribollet, Electrochemical Impedance Spectroscopy, Wiley, 2011.

[3] T. Paillat, G. Touchard, Electrical charges and liquids motion, Journal of Electrostatics. 67 (2009) 326-334. https://doi.org/10.1016/j.elstat.2009.01.038.

[4] A.P. Washabaugh, M. Zahn, Flow electrification measurements of transformer insulation using a Couette flow facility, IEEE Transactions on Dielectrics and Electrical Insulation. 3 (1996) 161-181. https://doi.org/10.1109/94.486767.

[5] X. Zhang, M. Zahn, Kerr electro-optic field mapping study of the effect of charge injection on the impulse breakdown strength of transformer oil, Applied Physics Letters. 103 (2013) 162906. https://doi.org/10.1063/1.4826185.

[6] M. Zahn, M. Hikita, K.A. Wright, C.M. Cooke, J. Brennan, Kerr Electro-Optic Field Mapping Measurements in Electron Beam Irradiated Polymethylmethacrylate, IEEE Transactions on Electrical Insulation. EI-22 (1987) 181-185. https://doi.org/10.1109/TEI.1987.298879.

[7] O. Stern, Zur theorie der elektrolytischen doppelschicht, Zeitschrift Für Elektrochemie Und Angewandte Physikalische Chemie. $30 \quad$ (1924) 508-516. https://doi.org/10.1002/bbpc.192400182.

[8] R.J. Fleming, Space charge profile measurement techniques: recent advances and future directions, IEEE Transactions on Dielectrics and Electrical Insulation. 12 (2005) 967-978. https://doi.org/10.1109/TDEI.2005.1522190.

[9] A. Imburgia, R. Miceli, E.R. Sanseverino, P. Romano, F. Viola, Review of space charge measurement systems: acoustic, thermal and optical methods, IEEE Transactions on Dielectrics and Electrical Insulation. 23 (2016) 3126-3142. https://doi.org/10.1109/TDEI.2016.7736878.

[10] M. Hao, Y. Zhou, G. Chen, G. Wilson, P. Jarman, Space charge behaviour in oil and impregnated pressboard combined insulation system, in: 2014 IEEE 18th International Conference on Dielectric Liquids (ICDL), 2014: pp. 1-4. https://doi.org/10.1109/ICDL.2014.6893154.

[11] B. Huang, M. Hao, J. Hao, J. Fu, Q. Wang, G. Chen, Space charge characteristics in oil and oil-impregnated pressboard and electric field distortion after polarity reversal, IEEE Transactions on Dielectrics and Electrical Insulation. 23 (2016) 881-891. https://doi.org/10.1109/TDEI.2015.005413.

[12] J.P. Jiang, B.X. Du, X.L. Li, B. Cui, H. Xu, and, Space charge measurement of vegetable oil filled with BN nanoparticles by thermal step method, in: 2016 IEEE International Conference on Dielectrics (ICD), 2016: pp. 176-179. https://doi.org/10.1109/ICD.2016.7547573.

[13] C. Cheng, Y. Wu, Y. Fan, K. Wu, K. Qian, Y. Liu, Correlation between Aging Status and Space Charge Behaviors in Samples Consisting of Oil Immersed Paper and Oil, in: 2018 IEEE Conference on Electrical Insulation and Dielectric Phenomena (CEIDP), 2018: pp. 614-617. https://doi.org/10.1109/CEIDP.2018.8544852.

[14] P.D.S. Clermont, T. Paillat, P. Leblanc, Numerical study on the impact of a temperature step on the electrical double layer, Journal of Electrostatics. 95 (2018) 13-23. https://doi.org/10.1016/j.elstat.2018.07.006.

[15] X. Sidambarompoulé, J. Laurentie, P. Notingher, T. Paillat, P. Leblanc, Assessment of Fallouts of the Thermal Step Method for measuring Space Charge in Liquid Dielectrics, 
in: 2018 IEEE 2nd International Conference on Dielectrics (ICD), 2018: pp. 1-4. https://doi.org/10.1109/ICD.2018.8514661.

[16] X. Sidambarompoulé, J. Laurentie, P. Notingher, T. Paillat, P. Leblanc, G. Touchard, A. Toureille, O. Guille, A Non-destructive Thermal Stimulus Method as a Tool for studying the Electrical Double Layer, in: 2019 IEEE 20th International Conference on Dielectric Liquids (ICDL), 2019: pp. 1-4. https://doi.org/10.1109/ICDL.2019.8796837.

[17] P. Leblanc, P.D.S. Clermont, T. Paillat, X. Sidambarompoulé, J. Laurentie, P. Notingher, Numerical Study of the Thermal Excitation Applied to a Dielectric Liquid Film, in: 2019 IEEE 20th International Conference on Dielectric Liquids (ICDL), 2019: pp. 1-4. https://doi.org/10.1109/ICDL.2019.8796727.

[18] X. Sidambarompoulé, Étude expérimentale et numérique sur l'utilisation d'un stimulus thermique pour la mesure de charges d'espace dans des liquides diélectriques. Application à la Double Couche Électrique, PhD Thesis, Université de Montpellier, 2020.

[19] A. Ndour, S. Holé, P. Leblanc, T. Paillat, Direct observation of electric charges at solid/liquid interfaces with the pressure-wave-propagation method, Journal of Electrostatics. 109 (2021) 103527. https://doi.org/10.1016/j.elstat.2020.103527.

[20] A. Toureille, G. Touchard, T. Richardson, Electrical Double Layer at Dielectric Liquid/Solid Interface. Space Charge measurements using the Thermal Step methods, in: Conference Record of the 1996 IEEE International Symposium on Electrical Insulation, 1996: pp. 758-762. https://doi.org/10.1109/ELINSL.1996.549455.

[21] A. Toureille, Measurement of Space Charges using the Thermal Step Method-Different Techniques for Numerical and Experimental Validation, Journal of Electrostatics. 32 (1994) 277-286. https://doi.org/10.1016/0304-3886(94)90016-7.

[22] J. Santana, Mesure de charges d'espace dans les câbles de transport de l'énergie électrique, PhD Thesis, 1994.

[23] A. Cernomorcenco, N. Jr P., Application of the thermal step method to space charge measurements in inhomogeneous solid insulating structures: A theoretical approach, Applied Physics Letters. 93 (2008). https://doi.org/10.1063/1.3005425.

[24] P. Notingher, S. Agnel, A. Toureille, Thermal step method for space charge measurements under applied dc field, IEEE Transactions on Dielectrics and Electrical Insulation. 8 (2001) 985-994. https://doi.org/10.1109/94.971455.

[25] M. Abou-Dakka, S. Bamji, A. Bulinski, Space-charge distribution in XLPE by TSM, using the inverse matrix technique, IEEE Transactions on Dielectrics and Electrical Insulation. 4 (1997) 314-320. https://doi.org/10.1109/94.598288.

[26] S. Holé, Resolution of direct space charge distribution measurement methods, IEEE Transactions on Dielectrics and Electrical Insulation. 15 (2008) 861-871. https://doi.org/10.1109/TDEI.2008.4543124.

[27] P. Notingher, A. Toureille, S. Agnel, J. Castellon, Determination of Electric Field and Space Charge in the Insulation of Power Cables With the Thermal Step Method and a New Mathematical Processing, IEEE Transactions on Industry Applications. 45 (2009) 67-74. https://doi.org/10.1109/TIA.2008.2009612.

[28] G. Touchard, Étude du transport de Charges dans les liquides Diélectriques, PhD Thesis, Université de Poitiers, 1978.

[29] T. Paillat, E. Moreau, G. Touchard, Space charge density at the wall in the case of heptane flowing through an insulating pipe, Journal of Electrostatics. 53 (2001) 171-182. https://doi.org/10.1016/S0304-3886(01)00139-5.

[30] M. Zdanowski, Streaming Electrification Phenomenon of Electrical Insulating Oils for Power Transformers, Energies. 13 (2020) 3225. https://doi.org/10.3390/en13123225. 
[31] A.A. Boumans, Streaming currents in turbulent flows and metal capillaries: II. Theory (2). Charge transported by the flow of liquid, Physica. 23 (1957) 1027-1037. https://doi.org/10.1016/S0031-8914(57)95960-8.

[32] F. Schlegel, Using the Boussinesq Approximation for Natural Convection, Comsol Blog. (2015). https://www.comsol.com/blogs/using-the-boussinesq-approximation-for-naturalconvection/. 